\title{
Coinfection with dengue and hepatitis A complicated with infective endocarditis in a Yemeni patient: a case report
}

\author{
Shafiq A. Alemad ${ }^{1}$, Abdulsalam M. Halboup ${ }^{2^{*}}$ (1), Khaled Aladeeb ${ }^{1}$, Mohamed Al-Saleh $^{1}$ and Nuha Al-Kufiley ${ }^{1}$
}

\begin{abstract}
Introduction: Coinfection with dengue and hepatitis A is rare and challenging for physicians since their clinical features can be overlapping. These infections are self-limiting but can become complicated by subsequent infective endocarditis. We report a case of infective endocarditis following a coinfection with dengue and hepatitis A.

Case presentation: A 17-year-old Yemeni male patient was admitted to the hospital complaining of yellowish discoloration of the skin and sclera associated with dark urine and a diffuse skin rash on the trunk and upper limbs followed by intermittent high-grade fever. Coinfection was confirmed by hepatitis A immunoglobulin $\mathrm{M}$ and dengue immunoglobulin M. At the time of diagnosis, white blood cells were normal, with mild neutrophilia and thrombocytopenia along with elevated C-reactive protein. Five days later, the patient was readmitted to the emergency department, complaining of high-grade fever, fatigue, myalgia, nausea, and vomiting. A systolic heart murmur was heard, and infective endocarditis was confirmed by the visualization of two vegetations on the mitral valve and coagulase-negative staphylococci after blood culture. Supportive therapies were initiated for hepatitis $\mathrm{A}$ and dengue fever, whereas infective endocarditis was treated with antibiotics for 4 weeks. The patient recovered completely from dengue, hepatitis $\mathrm{A}$, and infective endocarditis.

Conclusion: In endemic areas, it is reasonable to screen for coinfection with dengue and hepatitis A since they are superimposed on each other. Subacute infective endocarditis may occur following initial dengue and hepatitis A coinfection, especially among patients with rheumatic heart disease. An echocardiogram is a pivotal workup for evaluating a patient with persistent fever of unknown origin.
\end{abstract}

Keywords: Infective endocarditis, Dengue, Hepatitis A, Coagulase-negative staphylococci, Yemen

\section{Introduction}

Dengue is a public health concern in more than 100 countries worldwide [1]. According to World Health Organization (WHO) guidelines, dengue severity is classified into dengue with/without warning signs and severe dengue [2]. Dengue is endemic in Yemen, with reports on

\footnotetext{
*Correspondence: a_halboob@yahoo.com

2 Departments of Clinical Pharmacy and Pharmacy Practice, Faculty

of Pharmacy, University of Science and Technology, P.O. Box 13064, Sana'a, Yemen

Full list of author information is available at the end of the article
}

several outbreaks in Hodeidah, Taiz, Aden, and Hadhramout governorates [3-6]. People displacement, poor hygiene, and damaged health infrastructure due to the civil war are the main factors contributing to the spread of mosquito-borne diseases in the country $[6,7]$.

Coinfection with dengue is frequently caused by bacteria, followed by viruses [8]. There are several case reports on bacterial infections causing complications in patients with dengue. For instance, dengue can be complicated by Staphylococcus aureus causing pneumonia $[9,10]$ and abscess [11-13]. Additionally, staphylococcal original author(s) and the source, provide a link to the Creative Commons licence, and indicate if changes were made. The images or other third party material in this article are included in the article's Creative Commons licence, unless indicated otherwise in a credit line to the material. If material is not included in the article's Creative Commons licence and your intended use is not permitted by statutory regulation or exceeds the permitted use, you will need to obtain permission directly from the copyright holder. To view a copy of this licence, visit http://creativecommons.org/licenses/by/4.0/. The Creative Commons Public Domain Dedication waiver (http://creativeco mmons.org/publicdomain/zero/1.0/) applies to the data made available in this article, unless otherwise stated in a credit line to the data. 
endocarditis following classic and hemorrhagic dengue fever was also reported $[14,15]$.

Coinfection with dengue and hepatitis $\mathrm{A}$ in an individual is rare but challenging for medical professionals because of the overlap between their symptoms $[16,17]$. Moreover, bacteremia in a patient coinfected with dengue and hepatitis A is extremely rare and more challenging in terms of diagnosis and management, posing life-threatening complications. We report a case of an adult male who was initially diagnosed with dengue and hepatitis A coinfection but subsequently developed infective endocarditis by coagulase-negative staphylococci (CoNS).

\section{Case presentation}

A 17-year-old Yemeni male patient with rheumatic heart disease and a travel history to Hadhramout governorate in the east of the country was admitted to the hospital complaining of yellowish discoloration of the sclera and skin along with dark urine but without dysuria or frequency. Then, he developed an intermittent high-grade fever, headache, fatigue, myalgia, and abdominal discomfort together with food-related nausea, vomiting, and loss of appetite. On examination, the patient was febrile and had yellowish discoloration of the sclera along with a skin rash on his trunk and upper limbs. Systemic examination revealed bilateral basal lung crepitation along with mild tenderness and hepatomegaly, while cardiovascular and other systems were initially normal. The patient underwent tonsillectomy 10 years earlier and was on benzathine penicillin 6 months before admission. Socially, the patient was not a smoker, alcoholic, or drug-addicted.

The investigations performed on presentation (3 July 2019) to the Emergency Department revealed a normal white blood cell (WBC) count of $10.6 \times 10^{9} / \mathrm{L}$ (normal range: $4-11 \times 10^{9} / \mathrm{l}$ ), with a slightly increased neutrophil percentage to $83 \%$ (normal range $40-75 \%$ ). Serum C-reactive protein (CRP) was $253 \mathrm{mg} / \mathrm{L}$ (normal level $<5 \mathrm{mg} / \mathrm{L}$ ). The platelet count decreased to $115 \times 10^{9} / \mathrm{L}$ but then gradually increased. Peripheral blood smear examination showed normocytic, normochromic red blood cells (RBCs) with normal morphology of all cell types. Serum albumin concentration was $27.4 \mathrm{~g} / \mathrm{L}$ (normal 35-45 g/L), and urine was positive for bilirubin but did not contain RBCs and pus cells. Alanine aminotransferase level was $401 \mathrm{U} / \mathrm{L}$ (normal < $50 \mathrm{U} / \mathrm{L}$ ), while aspartate aminotransferase, alkaline phosphatase, and serum creatinine were within the normal range. Direct bilirubin concentration was $6.6 \mathrm{mg} / \mathrm{dL}$ (normal $<0.3 \mathrm{mg} / \mathrm{dL}$ ) and increased to $8.96 \mathrm{mg} / \mathrm{dL}$ on the readmission day (8 July 2019) but then decreased gradually to $1.65 \mathrm{mg} / \mathrm{dL}$ on discharge (17 July 2019). International normalized ratio and prothrombin time were 1.69 and 20.4 seconds (normal 13.5 seconds), respectively. The other investigations done during clinic visits, readmission, and before discharge are summarized in Table 1.

The clinical diagnosis of acute liver injury as a result of possible dengue and hepatitis coinfection was made based on the travel history to Hadhramout governorate, clinical presentation, and clinical examination. Both viral infections were confirmed by dengue immunoglobulin $M$ (IgM) and hepatitis A IgM antibodies test using enzymelinked immunosorbent assay (16 and 11.66; negative results for dengue and hepatitis $\mathrm{A}$ are less than 9 and 0.8 , respectively) on admission (3 July 2019). Tests for malaria and brucellosis were negative.

In the emergency department, supportive therapies for both hepatitis A and dengue were commenced using intravenous fluids, antipyretics, and antiemetic. Then, the patient was discharged home on intravenous dextrose saline, paracetamol $1 \mathrm{~g}$ four times a day as needed, and azithromycin $500 \mathrm{mg}$ once daily for 3 days. Azithromycin was given for suspected bacterial infection.

Five days later, the patient was readmitted (8 July 2019, the first day of readmission) to the Medical Male Ward complaining of high-grade fever not relieved by simple antipyretics associated with nausea and vomiting, malaise, myalgia, and decreased appetite. His heart rate and blood pressure were 112 beats/minute and $100 / 60 \mathrm{mmHg}$, respectively. The total WBC count increased to $21.77 \times 10^{9} / \mathrm{L}$, with $93.4 \%$ of the cells being neutrophils, while CRP was $67 \mathrm{mg} / \mathrm{L}$ on the first day and increased to $140 \mathrm{mg} / \mathrm{L}$ on the second day of illness.

Parenteral empirical therapy using meropenem (500 $\mathrm{mg}$ three times a day) with moxifloxacin $(400 \mathrm{mg}$ once a day) was started on the readmission day, and vancomycin ( $1 \mathrm{~g}$ twice a day) was added on the third day (10 July 2019) after a noticeable increase in the CRP levels. Further clinical examinations and laboratory investigations were performed to identify the source of infection. On examination, there was a systolic murmur (grade 3 ) at the mitral area and sacral edema on his back. The patient's chest echocardiogram revealed moderate mitral regurgitation and two vegetations on the mitral valve. The large one $(24 \times 11 \mathrm{~mm})$ attached to the anterior leaflet of the mitral valve, while the small one $(2.1 \times 0.9 \mathrm{~cm})$ attached to the posterior leaflet of the mitral valve as shown in Fig.1A, B. There was also mild tricuspid regurgitation with moderate pulmonary hypertension. The estimated ejection fraction by M-mode was $72 \%$. Other valves and the dimensions of the cardiac chambers were normal with no pericardial effusion. On the same day, the chest echocardiogram was repeated and showed the same findings as the first day. According to the modified Duke criteria, the diagnosis of infective endocarditis was made based on one major criterion (presence of vegetation) and two minor criteria (single positive culture and 
Table 1 Investigation details of the reported case

\begin{tabular}{|c|c|c|c|c|c|c|}
\hline Parameters (test) & $\begin{array}{l}\text { Findings } \\
\text { on first } \\
\text { admission } \\
(3 / 7 / 2019)\end{array}$ & $\begin{array}{l}\text { Finding after } \\
\text { discharge } \\
(6 / 7 / 2019)\end{array}$ & $\begin{array}{l}\text { Finding on } \\
\text { readmission } \\
(8 / 7 / 2019)\end{array}$ & $\begin{array}{l}\text { Findings on first } \\
\text { visit }(20 / 7 / 2019)\end{array}$ & $\begin{array}{l}\text { Findings on } \\
\text { second visit } \\
(27 / 7 / 2019)\end{array}$ & Normal value \\
\hline Hemoglobin (g/dL) & 13.6 & 14.6 & 11.9 & 11.0 & 10.7 & $13-18$ \\
\hline WBC (cells $/ \mathrm{mm}^{3}$ ) & 10.6 & 10.1 & 21.77 & 5.9 & 6.4 & $4-11 \times 10^{9} / \mathrm{L}$ \\
\hline Neutrophil & 83 & 69 & 93.4 & 53 & 50.0 & $40-70 \%$ \\
\hline Lymphocyte & 12 & 26 & 3.2 & 42 & 45.9 & $20-45$ \\
\hline Platelets (cells/mm³) & 115 & 135 & 168 & 432 & 377 & $150-450$ \\
\hline Total bilirubin (mg/dL) & & 11.11 & 9.843 & & & $0.1-1.2 \mathrm{mg} / \mathrm{dL}$ \\
\hline Direct bilirubin(mg/dL) & 6.6 & 10.7 & 8.96 & 1.92 & 1.22 & $<0.3$ \\
\hline SGOT (AST) & 40 & 44.0 & - & - & - & $<50$ \\
\hline SGPT (ALT) & 401 & 198 & 154 & 43 & 43.0 & Male $<50$ \\
\hline Alkaline Phosphatase (U/L) & 144 & 268 & - & - & - & Male $<270$ \\
\hline Hepatitis B surface antigen (HBSAg) & Negative & - & - & - & - & Negative $<1$ \\
\hline $\begin{array}{l}\text { C-reactive protein (CRP) by immuno- } \\
\text { turbidimetry }\end{array}$ & 253 & 14.0 & 67 & 3.92 & 1.45 & Negative $<5 \mathrm{mg} / \mathrm{L}$ \\
\hline ESR (mm/hour) & - & & - & - & 50 & Up to $10 \mathrm{~mm} / \mathrm{hour}$ \\
\hline Serum albumin (g/L) & 27.4 & 31.9 & - & 39.9 & - & $35-45$ \\
\hline Total protein (g/L) & & 80.3 & & & & Adult: $68-87$ \\
\hline Serum creatinine $(\mu \mathrm{mol} / \mathrm{L})$ & 78.69 & & & 124 & 110 & Adult: $<106$ \\
\hline Dengue virus IgM by (ELISA) & 16 & - & - & - & - & Negative $<9$ \\
\hline Hepatitis A IgM antibody & 11.66 & - & - & - & - & Negative $<0.8$ \\
\hline $\begin{array}{l}\text { Brucella total antibody titer by } \\
\text { agglutination }\end{array}$ & Negative & - & - & - & - & $<1: 80$ \\
\hline Prothrombin time (seconds) & 20.4 & 19.0 & 18.3 & 13.8 & - & Control: 13.5 \\
\hline International normalized ratio (INR) & 1.69 & 1.51 & 1.47 & 1.06 & - & - \\
\hline Malaria test & $\begin{array}{l}\text { Blood smear: } \\
\text { no malaria } \\
\text { seen } \\
\text { Rapid test } \\
\text { (antigen): } \\
\text { negative }\end{array}$ & - & - & - & - & - \\
\hline
\end{tabular}

Bold font represents normal laboratory values

WBC white blood cells, ALT alanine aminotransferase, AST aspartate aminotransferase, ESR erythrocyte sedimentation rate, ELISA enzyme-linked immunosorbent assay

fever). On the fourth day of hospitalization, a single blood culture yielded CoNS sensitive to ampicillin-sulbactam, cefoperazone-sulbactam, ceftriaxone-tazobactam, ciprofloxacin, clindamycin, doxycycline, gentamicin, imipenem, levofloxacin, lincomycin, moxifloxacin, and vancomycin, but it was resistant to amoxicillin/clavulanic acid and co-trimoxazole. At this time, meropenem and moxifloxacin were discontinued, and the patient was treated with ceftriaxone/sulbactam (1.5 g twice a day for 4 weeks) with a daily dose of gentamicin $(240 \mathrm{mg})$ with $100 \mathrm{ml}$ normal saline over 1 hour for 2 weeks.

On the seventh day of hospitalization, the patient complained of diarrhea (four times per day) for which upper abdominal ultrasound and Clostridium difficile antigen were requested. Ultrasound showed moderate hepatomegaly $(16 \mathrm{~cm})$ with mild bilateral pleural effusion, while
C. difficile antigen test was negative. Therefore, the treatment plan was not modified.

Treatment progress was monitored through the daily investigation of CRP, WBCs, and alanine aminotransferase. The patient was discharged on the tenth day of hospitalization after revealing a marked improvement on clinical and laboratory investigations. Other medications were added to the above-mentioned antibiotics, including bisoprolol (5 $\mathrm{mg}$ tablet once daily for 1 month) to control tachycardia, paracetamol (500 mg tablet for fever) as needed, furosemide (40 mg tablet once daily in the morning for 1 month) along with metolazone (1 mg tablet for 10 days) for pleural effusion and sacral edema. No additional echocardiogram was performed after discharge. During the first and second follow-up visits to the medical clinic, on day 3 and day 10 after discharge, the clinical features and laboratory investigations of the 


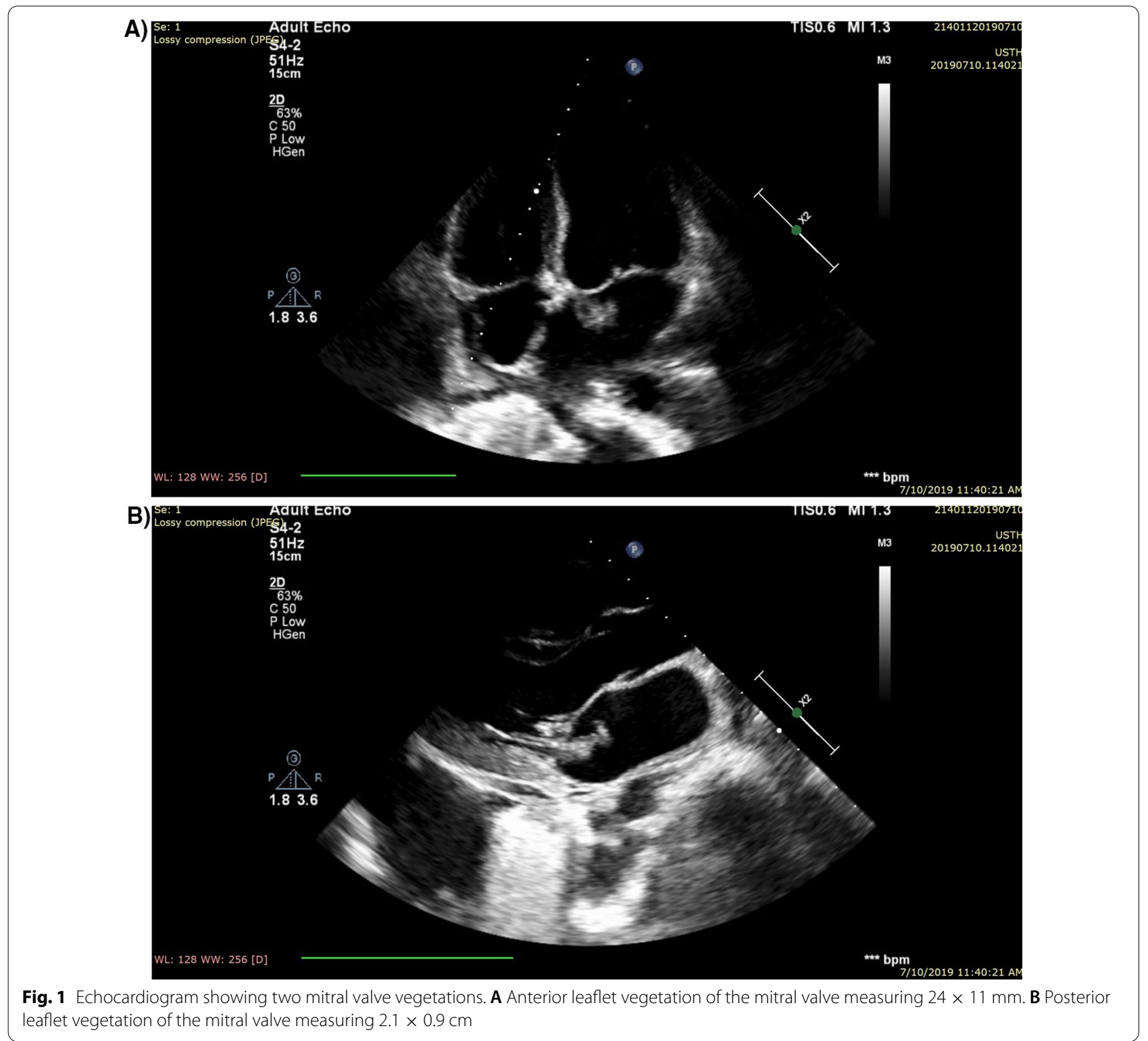

patients showed obvious improvement as summarized in Table 1.

\section{Discussion}

We report a case of infective endocarditis diagnosed a few days after the diagnosis and management of coinfection with dengue and hepatitis A in a 17-year-old Yemeni male patient. Although dengue and hepatitis A were diagnosed before infective endocarditis, we assumed that the latter coexisted with both infections but remained undiagnosed. This hypothesis is in agreement with the fact that our patient had a risk factor (rheumatic heart disease), was subjected to tonsillectomy 10 years earlier, and was on benzathine penicillin G (1.2 million units every 3 weeks) until 6 months before admission. The diagnosis of infective endocarditis was not established at the time of dengue and hepatitis A diagnosis because of several factors. These include the inability to perform blood cultures and echocardiogram at the first admission of viral coinfection since the patient was admitted and discharged home from the emergency department on the same day based on his request. Additionally, the long delay in the diagnosis of CoNS-induced infective endocarditis could be related to its indolent, mild, and prolonged disease course $[18,19]$. Furthermore, infective endocarditis is easily overlooked in a patient coinfected with dengue and viral hepatitis because of the overlap between 
several clinical features of such diseases that can pose a diagnostic dilemma. Triple infections are also sparsely reported in the literature.

Bacterial infections are the most frequent type of dengue-associated coinfection. Several mechanisms have been proposed to explain the increased susceptibility to secondary bacterial infection following dengue. One mechanism is the damage to the endothelial tissue of the endocardium, predisposing to bacteria colonization and endocarditis during skin breaching by cannula [20]. Another mechanism is the dysregulation of the immune system caused by high levels of interleukin-10 among dengue patients, predisposing them to secondary bacterial infection [21].

Several studies have described multiple infections in patients with dengue. Patients with severe dengue [10], prolonged fever (more than 5 days), with or without acute renal failure [22], increased WBC count, decreased lymphocytes [23], or hyponatremia [24] are at high risk for concurrent bacterial infection. Most of these factors predispose a patient to develop infective endocarditis. In our case, several risk factors, including prolonged fever, leukocytosis, decreased lymphocytes, increased CRP, high pulse rate, and hyponatremia along with hepatic failure, predispose him to develop infective endocarditis.

Staphylococcal infections following classic or hemorrhagic dengue were reported in patients with thyroid abscess [11], intramuscular abscesses [12, 13], infective endocarditis $[14,15,25]$, and pneumonia $[9,10]$. Furthermore, CoNS were isolated from hospitalized adults with dengue [26]. In our case, skin breaches occurring during cannulation might have facilitated the entry of CoNS into his blood circulation, leading to bacteremia and subsequent infective endocarditis (Additional file 1, Additional file 2).

Coinfection with dengue and hepatitis $\mathrm{A}$ has been reported in several case reports [16, 17, 27-30]. Overlapping of clinical features between dengue and hepatitis A can lead to substantial misdiagnosis. Therefore, it is reasonable to screen for both types of infection in endemic areas. In our case, the marked increase in serum aminotransferase levels, deranged prothrombin time, and jaundice were common features of acute viral hepatitis, while thrombocytopenia, hemoconcentration, hepatomegaly, abdominal tenderness, and third spacing were common features of dengue with warning signs.

\section{Conclusion}

In endemic areas, coinfection with dengue and viral hepatitis can be encountered among young adults with rheumatic heart disease. A patient presenting with elevated liver enzymes, deranged coagulation profile, prolonged fever, pleural effusion, and thrombocytopenia should alert clinicians towards dengue and hepatitis A coinfection. Highly elevated aminotransferase levels (eight to ten times the upper limit) are consistent with acute viral hepatitis compared with two to three times in dengue fever, and the ratio of aspartate aminotransferase to lactate dehydrogenase is more than 4 in acute hepatitis. Nevertheless, elevated CRP and high pulse rate along with prolonged and high-grade fever in a patient with dengue should be considered as alarming signs for concurrent bacterial infection. Therefore, echocardiography and blood culturing are pivotal workups in patients with persistent fever of unknown origin. Moreover, timely administration of optimal antibiotics is critical for patients with mixed infections to avoid life-threatening complications.

This study has some limitations. One of the limitations is that the specific pathogen of CoNS that induced endocarditis in this patient was not determined due to a resource-limited setting. The second important limitation is that blood samples for cultures were collected after the administration of empirical antibiotics. One more limitation is that several laboratory investigations were not requested during hospitalization since the patient was not covered by health insurance.

\section{Abbreviations \\ CoNS: Coagulase-negative staphylococci; CRP: C-reactive protein; WBC: White blood cells; RBCs: Red blood cells.}

\section{Supplementary Information}

The online version contains supplementary material available at https://doi. org/10.1186/s13256-021-03069-w.

Additional file 1. The video shows anterior leaflet vegetation.

Additional file 2. The video shows posterior leaflet vegetation.

\section{Acknowledgements}

We would like to express our thanks to the patient and his family. Also, we want to thank Dr. Rashad Abdul-Ghani for his great effort in improving the quality of the manuscript.

\section{Authors' contributions}

SAA supported the study design and review the final draft. AMH supported the study design and helped in manuscript drafting. KA was responsible for reviewing the manuscript and provided us with echocardiogram results and labeling. MA and NA helped in manuscript reviewing and patient follow-up. All authors were involved in case management. All authors read and provided approval for publication.

Funding

There was no funding for this case report

\section{Availability of data and materials}

The data that support the findings of this case report are available from the medical records of the male medical department at University of Science and Technology Hospital, Sana'a, Yemen. 


\section{Declarations}

Ethical approval and consent to participate

This study was approved by Ethics Committee of University of Science and Technology (EAC/UST 195)

\section{Consent for publication}

Written informed consent was obtained from the patient's father for publication of this case report and any accompanying images. A copy of the written consent is available for review by the Editor-in-Chief of this journal

\section{Competing interests}

The authors declare that they have no competing interests

\section{Author details}

'University of Science and Technology Hospital, Sana'a, Yemen. ${ }^{2}$ Departments of Clinical Pharmacy and Pharmacy Practice, Faculty of Pharmacy, University of Science and Technology, P.O. Box 13064, Sana'a, Yemen.

Received: 4 June 2020 Accepted: 18 August 2021

Published online: 11 September 2021

\section{References}

1. Guha-Sapir D, Schimmer B. Dengue fever: new paradigms for a changing epidemiology. Emerg Themes Epidemiol. 2005;2(1):1. https://link.springer. com/article/https://doi.org/10.1186/1742-7622-2-1

2. Organization WH, Research SPf, Diseases TiT, Diseases WHODoCoNT, Epidemic WHO, Alert P. Dengue: guidelines for diagnosis, treatment, prevention and control: World Health Organization; 2009.

3. Madani TA, Abuelzein E-TM, Al-Bar HM, et al. Outbreak of viral hemorrhagic fever caused by dengue virus type 3 in Al-Mukalla, Yemen. BMC infect Dis.2013;13(1):136. DOl: https://doi.org/10.1186/1471-2334-13-136

4. Rezza G, El-Sawaf G, Faggioni G, et al. Co-circulation of dengue and chikungunya viruses, Al Hudaydah, Yemen, 2012. Emerg Infect Dis. 2014;20(8):1351. https://www.ncbi.nlm.nih.gov/pmc/articles/PMC41 11199/

5. Mohamed NA, El-Raoof EA, Ibraheem HA. Respiratory manifestations of dengue fever in Taiz-Yemen. Egyptian Journal of Chest Diseases and Tuberculosis. 2013;62(2):319-23. https://www.sciencedirect.com/science/ article/pii/S0422763813000289

6. Alghazali KA, Teoh B-T, Loong S-K, et al. Dengue outbreak during ongoing civil war, Taiz, Yemen. Emerg Infect Dis. 2019;25(7):1397. https://doi.org/ 10.3201/eid2507.180046.

7. Alghazali KA, Teoh B-T, Sam S-S, et al. Dengue fever among febrile patients in Taiz City, Yemen during the 2016 war: clinical manifestations, risk factors, and patients knowledge, attitudes, and practices toward the disease. One Health. 2020;9: 100119. https://doi.org/10.1016/j.onehlt. 2019.100119

8. Panda PK, Mohta S, Wig N, Soneja M. Dengue co-infections-an emerging entity during the outbreak. J Clin Diagn Res.2018;12(4).. DOI: https://doi. org/10.7860/JCDR/2018/32002.11385

9. Miyata N, Yoshimura Y, Tachikawa N, Amano Y, Sakamoto Y, Kosuge Y. Cavity forming pneumonia due to Staphylococcus aureus following dengue fever. The Am J Trop Med Hyg. 2015;93(5):1055-7. https://doi.org/10. 4269/aitmh.15-0045.

10. Nagassar RP, Bridgelal-Nagassar RJ, McMorris N, Roye-Green KJ. Staphylococcus aureus pneumonia and dengue virus co-infection and review of implications of coinfection. BMJ Case Rep. $2012 \mathrm{Jul}$ 3;2012:bcr0220125804. PubMed PMID: 22761204. Pubmed Central PMCID: 3391412 . https://www.ncbi.nlm.nih.gov/pubmed/22761204

11. Meena DS, Kumar D, Bohra GK, et al. Concurrent methicillin-resistant Staphylococcus aureus septicemia and thyroid abscess in a young male with dengue. Infect Disord Drug Targets. 2020. https://doi.org/10.2174/ 1871526520666200312160701.

12. Huang S-H, Chung KM, Chen C-C, et al. Concomitant Staphylococcus aureus intra-muscular abscess after dengue hemorrhagic fever-a case report. 臺灣整形外科醫學會雜誌. 2016;25(1):58-63.
13. Ehelepola N, Rajapaksha R, Dhanapala D, Thennekoon T, Ponnamperuma S. Concurrent methicillin-resistant Staphylococcus aureus septicemia and pyomyositis in a patient with dengue hemorrhagic fever: a case report. BMC infect Dis. 2018;18(1):99. https://doi.org/10.1186/s12879-018-3012-1.

14. Samarasinghe $H$, Indrakumar J. Acute staphylococcal endocarditis following dengue haemorrhagic fever. J Ceylon Coll Physicians. 2012;43:54-5. https://doi.org/10.4038/jccp.v43i1-2.6819.

15. Araújo SA, Moreira DR, Veloso JMR, Silva JO, Barros VLSR, Nobre V. Fatal staphylococcal infection following classic dengue fever. Am J Trop Med Hyg. 2010;83(3):679-82. https://doi.org/10.4269/ajtmh.2010.10-0009.

16. Dey A. Hepatitis A and dengue coinfection. J Vector Borne Dis. 2015;52(3):265.. https://pubmed.ncbi.nIm.nih.gov/26418660/

17. Volchkova $E$, Umbetova K, Belaia $\mathrm{O}$, et al. Co-infection of dengue fever and hepatitis A in a Russian traveler. IDCases. 2016;5:67-8. https://doi.org/ 10.1016/j.idcr.2016.07.002

18. Becker K, Heilmann C, Peters G. Coagulase-negative staphylococci. Clin Microbiol Rev. 2014;27(4):870-926. https://cmr.asm.org/content/27/4/870

19. Rasmussen RV, Snygg-Martin U, Olaison L, et al. One-year mortality in coagulase-negative Staphylococcus and Staphylococcus aureus infective endocarditis. Scand J Infect Dis. 2009;41(6-7):456-61. https://doi.org/10. 1080/00365540902896061.

20. Lin C-F, Lei H-Y, Shiau A-L, et al. Endothelial cell apoptosis induced by antibodies against dengue virus nonstructural protein 1 via production of nitric oxide. J Immunol. 2002;169(2):657-64. https://doi.org/10.4049/ jimmunol.169.2.657.

21. Flores-Mendoza LK, Estrada-Jiménez T, Sedeño-Monge $V$, Moreno M, Manjarrez MDC, González-Ochoa G, Millán-Pérez Peña L, Reyes-Leyva J. $\mathrm{IL}-10$ and socs3 are predictive biomarkers of dengue hemorrhagic fever. Mediators Inflamm. 2017. https://doi.org/10.1155/2017/5197592.

22. Lee I-K, Liu J-W, Yang KD. Clinical characteristics and risk factors for concurrent bacteremia in adults with dengue hemorrhagic fever. T Am J Trop Med Hyg. 2005;72(2):221-6. https://pubmed.ncbi.nlm.nih.gov/15741560/

23. Thein T-L, Ng E-L, Yeang MS, Leo Y-S, Lye DC. Risk factors for concurrent bacteremia in adult patients with dengue. J Microbiol Immunol Infect. 2017;50(3):314-20. https://doi.org/10.1016/j.jmii.2015.06.008.

24. Trunfio M, Savoldi A, Viganò O, Monforte AdA. Bacterial coinfections in dengue virus disease: what we know and what is still obscure about an emerging concern. Infection. 2017;45(1):1-10. DOl: https://doi.org/10. 1007/s15010-016-0927-6

25. Samarasekara K, Munasinghe J. Dengue shock syndrome complicated with acute liver failure and kidney injury, infective endocarditis, and deep vein thrombosis: a case report. J Med Case Rep. 2018;12(1):1-4. https:// doi.org/10.1186/s13256-018-1862-1.

26. Syue L-S, Tang H-J, Hung Y-P, et al. Bloodstream infections in hospitalized adults with dengue fever: clinical characteristics and recommended empirical therapy. J Microbiol Immunol Infect. 2019;52(2):225-32. https:// doi.org/10.1016/j.jmii.2018.11.003.

27. Gavlak D. Health system in Yemen close to collapse: Yemen is facing a growing humanitarian catastrophe as health workers there risk their lives to help civilians caught up in the deadly conflict. Bull World Health Organ. 2015;93(10):670. https://www.who.int/bulletin/volumes/93/10/ 15-021015/en/

28. Gohal G. A fatal hepatitis A virus with dengue fever co-infection. IDCases. 2019;15:e00522. PubMed PMID: 30993077. Pubmed Central PMCID: 6441740. DOl: https://doi.org/10.1016/j.idcr.2019.e00522

29. Ranathunga I, Kannangara L, Bandara K, Gunatilake S. A rare presentation of coinfection: dengue virus and hepatitis A virus. Case Rep Infect Dis. 2019;2019. DOI: https://doi.org/10.1155/2019/9782892

30. Zaki S, Lad V. Concurrent infection of dengue fever and hepatitis A infection: a case report. Indian J Crit Care Med. 2011;15(4):238. https://doi.org/ 10.4103/0972-5229.92073.

\section{Publisher's Note}

Springer Nature remains neutral with regard to jurisdictional claims in published maps and institutional affiliations. 